\title{
Effect of Low-Level Laser Therapy on Muscle Strength and Endurance and Post-Exercise Recovery of Young Adult: A Double- Blind, Placebo-Controlled, Randomized Clinical Trial
}

\author{
${ }^{1}$ Amir Hosein Abedi Yekta, ${ }^{2}$ Faraj Tabeii, ${ }^{1}$ Shahin Salehi, ${ }^{3}$ Mohammad-Reza Sohrabi, \\ ${ }^{1}$ Mehrshad Poursaeidesfahani, ${ }^{4}$ Mohammad Hassabi", ${ }^{1}$ Nina Hazegh", ${ }^{1}$ Behnaz Mahdaviani \\ ${ }^{1}$ Department of Sports Medicine, Faculty of Medicine, Shahid Beheshti University of Medical Sciences, \\ Tehran, Iran. ${ }^{2}$ Department of Nuclear Medicine, Faculty of Medicine, Shahid Beheshti University of \\ Medical Sciences, Tehran, Iran. ${ }^{3}$ Department of Community Medicine, Faculty of Medicine, Shahid \\ Beheshti University of Medical Sciences, Tehran, Iran. ${ }^{4}$ Taleghani Hospital, Research Development \\ committee, Department of Sports Medicine, Shahid Beheshti University of medical Sciences, Tehran, Iran.
}

Submitted 29 July 2020; Accepted in final form 30 October 2020.

\begin{abstract}
Background. Recovery or post-exercise rehabilitation is an essential part of exercise training. Low-level Laser Therapy (LLLT) is a modality increasing interest for recovery because of having a conservative and non-aggressive method. LLLT also decreases the production of fatigue-related biomechanical markers, such as lactate, C-reactive protein (CRP), and Creatine kinase $(\mathrm{CK})$. Objectives. To investigate the effect of low-level laser therapy on muscle strength and endurance and post-exercise recovery of young adults between 20-35 years old. Methods. Fifty subjects were enrolled in this double-blind, placebo-controlled, randomized clinical trial study. Three days after the familiarization session, the subjects were divided into two groups via block randomization: the first group received pre-exercise laser at $810 \mathrm{~nm}, 60 \mathrm{~mW}$, and $60 \mathrm{~Hz}$ frequency for 30 seconds on three point's rectus femoris muscle. The other group received a placebo laser on the same points. The operator who was blinded to the allocation of the subjects applied the phototherapy. They underwent an exercise test with a treadmill and a maximum number of knee extensions with a 10 Kilograms (Kg) weight. The test results, level of blood lactate, perceived exertion of fatigue, and muscle soreness were assessed, and the results were analyzed. Results. The blood lactate level, the severity of muscle pain, the perceived exertion of fatigue, and the repetition of knee extensions with a 10 $\mathrm{kg}$ weight, had a statistically significant difference between laser and placebo groups $(\mathrm{p}<0.05)$. Conclusion. The use of LLL before exercise may improve muscular performance and reduce muscle soreness and fatigue.
\end{abstract}

KEYWORDS: Low-Level Laser Therapy (LLLT), Muscle Performance, Muscle Fatigue.

\section{INTRODUCTION}

Since the 1960-decade, Low-level laser therapy (LLLT) has been used for the healing of different injuries such as soft tissues (1-5), bones (6-8), and nervous tissues (9-14). In the last decade, several studies have investigated the positive effects of LLLT on muscle performance and fatigue (15-19). Recovery after exercise plays an essential role in injury prevention in sports medicine. Fatigue decreases the speed and the power of muscle contractions and the level of exercise performance $(15,16)$. It also increases the blood markers that are related to muscular damages, such as Creatine kinase (CK), C reactive protein (CRP), and Lactate dehydrogenase (LDH) (16-18). Therefore, postexercise recovery effectively increases pain relief,

*. Corresponding Authors:

First: Nina Hazegh, Assistant Professor; E-mail: h.anahita64@yahoo.com

Second: Mohammad Hassabi, Professor; E-mail: hassabi@yahoo.com 
inflammation subsidence, and acceleration of the repairing process in the damaged tissues (16). Different investigators have used various rehabilitation modalities, including NonSteroidal Anti-Inflammatory Drugs (NSAID), ultrasound, homeopathy, acupuncture, electrotherapy, massage, and cryotherapy (16, 17). Low-level laser therapy, LLLT, has been used as a new treatment to prevent injuries and control muscle soreness $(16,17)$. The suggested mechanism of LLLT action is the stimulation of the mitochondrial system and the effect on cytochrome $\mathrm{C}$ oxidase that enhances muscle power due to the acceleration of ATP production, leading to fatigue delay and protection of muscle against injury $(18,19)$. LLLT decreases the production of fatigue-associated markers such as LDH, CRP, and CK (19) and, by enhancing the oxidase system, reduces oxidative stress and elevates muscle buffering capacity (20-22).

Different investigators have studied the effects of LLLT on muscle performance and fatigue, suggesting various protocols regarding the wavelength, power density, irradiation time, and light sources of the laser, with an apparent inconsistency among the results (23). By considering previously reported results, this study aimed to investigate the effectiveness of the LLLT on muscle performance and fatigue among young adults. The wavelength of 810 nanometers (nm) used in this study is widely used in practice but has rarely been investigated in previous studies.

\section{MATERIALS AND METHODS}

Study Design and Protocol. Subjects who fulfilled the inclusion criteria were enrolled in the study were after signing informed consent and being fully informed about the study protocol. This trial is registered on the Iranian Registry of Clinical Trials (IRCT) website under the trial code IRCT20200415047097. The ethical committee of Shahid Beheshti University of Medical Sciences approves this trial under the ethical code IR.SBMU.MSP.REC.1397.459.

Subjects. The participants were fifty volunteers (31 male and 19 female) aged 20-35, referring to the sports medicine ward of Taleghani hospital during the year 2018 that met the inclusion criteria. Participants were enrolled in the trial after being invited and explained about the trial process by sport medicine residents and signing the informed consent.
Inclusion and Exclusion Criteria. The inclusion criteria were age in the range of 20-35, being sedentary (no history of regular exercise during the past three months), no history of professional sport during the lifetime, not consuming any drug or supplements, and no musculoskeletal disorder that prevents the use of treadmill or weight.

The exclusion criteria were being in the menstrual period in women, withdrawal from participating in the study

Familiarization Session. Three days before the experiment, the subjects underwent an exercise test with a treadmill and a maximum number of knee extensions with a 10 Kilograms $(\mathrm{Kg})$ weight.

Study Protocol. After explaining the subjects' study procedure and signing informed consent, subjects were enrolled in the trial. In the familiarization session, a form in which they were asked about demographic data, medical and activity history was filled by the participants. Then 5 minutes warm-up as treadmill walking with $2-3 \mathrm{~km} / \mathrm{h}$ was done.

Resistance Test. After warming up, a resistance test was done, as the maximum number of knee extensions in the full range of motion with a $10 \mathrm{Kg}$ weight machine (brand JK EXER, made in Taiwan) and the results (the maximum number of knee extensions) were recorded.

Aerobic Test. After the resistance test, the aerobic test was performed as a vigorous session of running on a treadmill (brand AVECINNA, made in Iran) with an intensity of $80-90 \%$ of the reserve heart rate for 15 minutes. Then cool-down phase as 5 minutes walking at $3-4 \mathrm{~km} / \mathrm{h}$ and hamstring stretching for 20 seconds were done. Subjects were asked not to have exercise at home for three days after the test.

Immediately after exercise, the blood lactate level was measured by a lactometer (brand ECF, made in Italy) and lactometer kit (brand Sensal, no 74 , made in China), using a fingertip blood sample. The blood sample was collected by puncturing the fingertip using a lancet (brand Ava, made in Iran). The Subjects were asked to rate their feeling of fatigue by RPE (Rating of Perceived Excretion) scale from 6 (minimal fatigue) to 20 (exhaustion). One hour later, the lactate test was repeated, and the results were recorded.

A telephone interview was done 24 hours later, and the subjects were asked about having any muscle soreness. If they felt any pain, they were asked to rate the severity of it from 1 (the 
least pain) to 10 (the most severe pain) By VAS (Visual Analog Scale) score.

Three days later, the subjects were randomly divided into two groups: the treatment group received one session of the real laser at $810 \mathrm{~nm}$ wavelength, $60 \mathrm{~mW}$ power, $60 \mathrm{~Hz}$ frequency by a laser device (brand MPTC, model LMPT 200, made in Iran) on 3 points of rectus femoris muscle, 30 seconds on each point (Figure 1), when the subject in the supine position. In the control group, the device was off, and a probe with no laser (placebo) on the same points was applied. As a landmark, the distance between the Anterior Superior Iliac Spine (ASIS) to the upper border of the patella was divided into 3 equal regions, and the middle point of every region was considered as an irradiation point. The aerobic and the resistance tests and evaluations were repeated. The results were measured and analyzed. Figure 2 indicates the study design.

Primary and Secondary Outcomes. The primary outcome was the maximum number of complete knee extensions with a $10 \mathrm{~kg}$ weight and the blood level of lactate (measured by the lactometer). The secondary outcome was fatigue (measured by RPE scale) and muscular pain (measured by VAS score).

Sample Size Calculation. The sample size was determined based on a previous study with a similar protocol, done by Feraressi et al. on 40 participants (24) with $\alpha=5 \%$ and $\beta=20 \%$ and effect size of $95 \% .25 \%$ of the sample size was added to it due to the probability of sample loss. The total number of the sample was set 50,25 in each group, and it was sufficient for this trial.

Randomization. Block randomization was performed to minimize the risk of selection bias. The participants, according to their Body Mass Index or BMI, were divided into two subgroups of BMI less than 25 (22 participants) and BMI equal to 25 or more (28 participants). Randomization was carried out in each subgroup separately, with a block size of 4.2 cards of $A$ and two cards of B were shuffled and randomly put together and the operator of the randomization, according to the order that the cards were arranged, assigned the participants to groups A or B. The participant who had received card A was placed in group A and received a real laser, whereas the participant who had received card B was placed in group B and received a placebo. The trial operators carried out all of the randomization steps. The participants and the operators of randomization did not know to which group A and B cards belong.

Blinding. Double-blinding was done to avoid performance bias, meaning that the participant did not know which group is settled. The operator was unable to differentiate between active and placebo laser. The laser device was programmed by a nurse who was not in contact with the participants. Then a second operator who was blinded to the allocation of the subjects applied the phototherapy. The laser device was where the operator could not see whether it is on or off.

Data Analysis. For statistical analysis, SPSS version 16 was used. Statistical tests as a t-test, Shapiro-Wilk test, and Wilcoxon test was also done on the data. A P-value of less than 0.05 was considered significant.

\section{RESULTS}

This study was performed on 50 sedentary participants in Taleghani Hospital, the sports medicine ward. Subjects were accidentally divided into two groups containing 25 persons as real or placebo laser groups (Table 1).

Table 1. Demographic Information of Participants

\begin{tabular}{lcc}
\hline & Treatment Group & Placebo Group \\
\hline Age & $22.92 \pm 4.06$ & $24.8 \pm 2.41$ \\
Gender & & \\
$\quad$ Male & $17(68 \%)$ & $14(56 \%)$ \\
$\quad$ Female & $8(32 \%)$ & $11(44 \%)$ \\
Height & $1.73 \pm 0.78 \mathrm{~m}$ & $24.4 \pm 3.66$ \\
Weight & $77.3 \pm 9.3$ & $72.09 \pm 6.62$ \\
Body Mass Index (BMI) & $25.4 \pm 1.95$ & $25.6 \pm 1.96$ \\
\hline
\end{tabular}

The Effect of Laser on Blood Lactate Level in Treatment and Placebo Groups. The paired t-test was used to assess the effect of laser on the lactate level in the $2^{\text {nd }}$ session immediately after exercise in both the placebo and treatment groups.
The result showed a significant difference between blood lactate level in real laser and placebo laser groups $(\mathrm{p}=0.018)$ (Table 2).

The Effect of Laser on the Number of Weight Repetitions in participants in Treatment and 
Placebo Groups. A paired t-test was used to assess the effect of laser on the maximum number of knee extensions with a $10 \mathrm{~kg}$ weight in participants in treatment and placebo groups, and the result showed a significant difference between real laser and placebo groups $(\mathrm{p}=0.018)($ Table 2$)$.

Table 2. Effect of Laser on Lactate Level, Weight Repetition, Fatigue and Muscle Pain in Both Treatment and Placebo Groups

\begin{tabular}{|c|c|c|c|c|c|c|}
\hline & Max & Min & Mean \pm SD & $\begin{array}{c}\text { P-Value } \\
\text { Shapirowilk Tst }\end{array}$ & $\begin{array}{c}\text { P-Value of } \\
\text { Paired T-Testt }\end{array}$ & $\begin{array}{c}\text { P-Value of } \\
\text { Wilcoxon Test }\end{array}$ \\
\hline $\begin{array}{l}\text { Lactate level in the } 2^{\text {nd }} \\
\text { session }\end{array}$ & & & & & 0.018 & \\
\hline Treatment & 17.41 & 4.76 & $11.23 \pm 3.43$ & 0.687 & & \\
\hline Placebo & 16.49 & 2.01 & $8.68 \pm 3.82$ & 0.58 & & \\
\hline $\begin{array}{l}\text { Weight repetition } 2^{\text {nd }} \\
\text { session }\end{array}$ & & & & & 0.018 & \\
\hline Treatment & 16 & 9 & $12.52 \pm 1.82$ & 0.162 & & \\
\hline Placebo & 16 & 8 & $11.36 \pm 2.41$ & 0.111 & & \\
\hline $\begin{array}{l}\text { Fatigue } \\
\text { perception(RPE) } 2^{\text {nd }} \\
\text { session }\end{array}$ & & & & & & P-value $<0.001$ \\
\hline Treatment & 14 & 8 & $10.96 \pm 1.24$ & 0.045 & & \\
\hline Placebo & 17 & 13 & $14.76 \pm 1.28$ & 0.048 & & \\
\hline $\begin{array}{l}\text { Muscle pain(VAS } \\
\text { score) } 2^{\text {nd }} \text { session }\end{array}$ & & & & & & P-value $<0.001$ \\
\hline Treatment & 5 & 2 & $3.12 \pm 0.83$ & 0.003 & & \\
\hline Placebo & 10 & 3 & $5.08 \pm 1.55$ & 0.008 & & \\
\hline
\end{tabular}

The Effect of Laser on Fatigue in Participants in Treatment and Placebo Groups. Fatigue perception was measured subjectively by each participant by using the RPE scale, scoring 6 (no fatigue) to 20 (exhaustion).

Wilcoxon test was used to assess the effect of laser on fatigue perception in participants in both groups in the $2^{\text {nd }}$ session, and the result showed a significant effect of laser on fatigue perception $(\mathrm{p}<0.001)$ (Table 2).

The Effect of Laser on Muscular Pain in Participants in Treatment and Placebo Groups. The effect of laser on muscular pain- so- called delayed onset muscle soreness (DOMS) in participants of treatment and placebo groups was assessed by telephone interview 24 hours after the exercise session. Subjects were asked about their pain sensation by the VAS scoring scale to grade their muscular pain from 1(no pain) to 10 (maximum of tolerable pain).

Wilcoxon test was used to assess muscular pain in the $2^{\text {nd }}$ session in both treatment and placebo groups. Results showed a significant difference between the two groups, meaning the laser effectively reduces muscle pain $(\mathrm{p}<0.001)$ (Table 2).

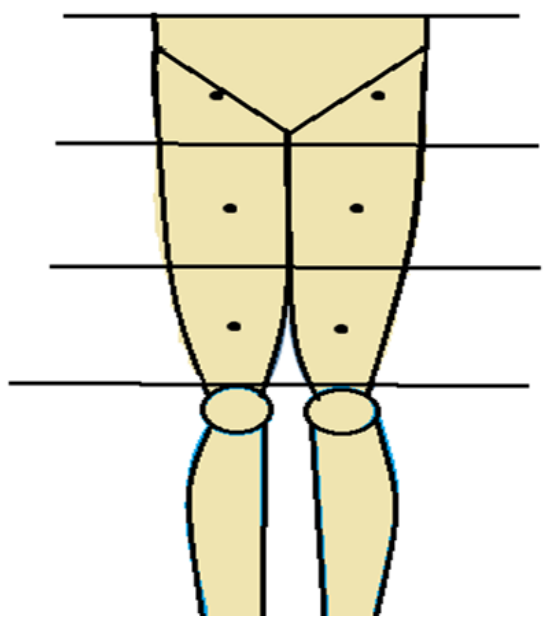

Figure 1. Laser Irradiation Points of Participants 


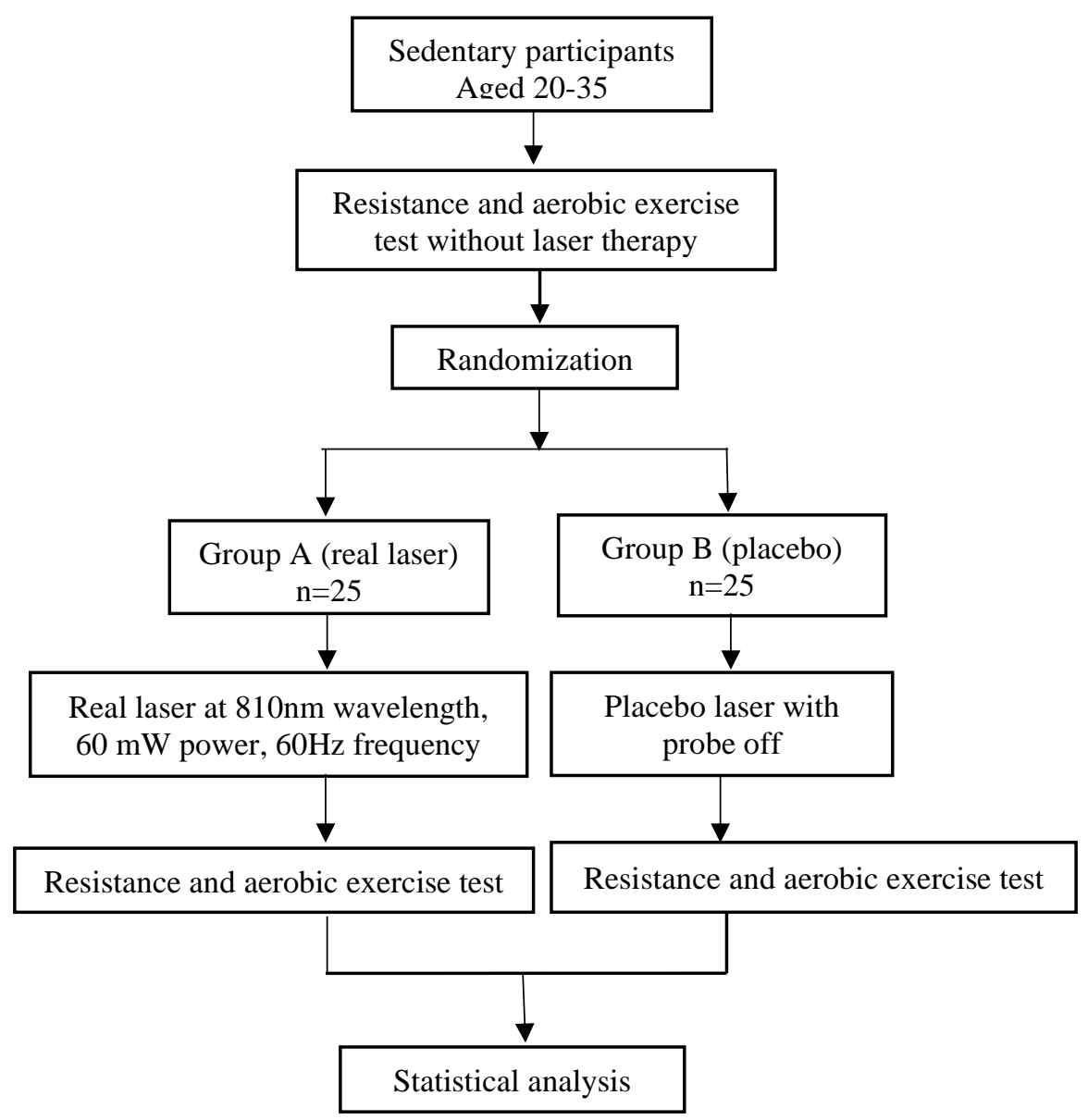

Figure 2. Flowchart of Study Design

\section{DISCUSSION}

Laser therapy is a part of a therapeutic system called Photobiomodulation, which is a technique of soft tissue treatment by using the energy of light waves (25). According to the required effect, various wavelengths starting from red $(630-660 \mathrm{~nm})$ to infrared $(808-950 \mathrm{~nm})$ spectrums are used in this technique. The infrared spectrum is more absorbable and has more therapeutic effects.

In this study, the blood lactate level was measured as one of the muscular injury indexes. The placebo group's level of lactate in both sessions immediately and 1 hour after exercise showed a significant difference. This finding indicates that time is sufficient on the blood lactate level.

The placebo group's lactate level did not show any significant difference immediately and one hour after exercise in the 2nd session.

Lactate level showed a significant difference between the first and $2^{\text {nd }}$ session immediately after exercise in the treatment group. A significant difference was seen between lactate level 1 hour after exercise in the first and seco $^{\text {nd }}$ sessions. These findings show that the laser is effective on the lactate level. In a study done by Ferraressy et al. on one professional athlete, the laser's effect at $850 \mathrm{~nm}$ on blood and urine $\mathrm{CK}$, aerobic capacity $\left(\mathrm{VO}_{2}\right)$, and blood lactate level as muscular fatigue markers were investigated. In this study, the laser was applied on the trunk and upper and lower limbs. They suggested using a laser before an exercise bout may decrease post-exercise blood lactate levels and improve renal and muscular performance (24). In a systematic review performed by Ferraressy et al. on 46RCTs, the authors demonstrated that the pre-exercise laser could reduce post-exercise lactate significantly (25).

In an investigation done in 2010 by Lealjunior et al., it was verified that using laser before exercise may decrease immediately post-exercise 
blood lactate level, but the level of blood lactate $5,10,15$, and 20 min after exercise did not show any significant differences between treatment and placebo groups (26).

In an investigation in 2017 done by Toma et al. by applying laser at $904 \mathrm{~nm}$ on 18 female volunteers, it was suggested that pre-exercise laser might reduce fatigue but does not make any significant changes in lactate level (22).

In this study, the placebo group's number of weight repetitions did not show any significant changes between the first and $\operatorname{seco}^{\text {nd }}$ groups. In contrast, this number showed a significant difference in the treatment group between the first and $\mathrm{seco}^{\text {nd }}$ sessions. Also, the number of weight repetitions in the $2^{\text {nd }}$ session of the placebo group and the treatment group showed a significant difference. These findings demonstrate laser to be effective in the improvement of muscular strength and endurance.

In a study in 2010 by Leal junior et al., it was proved that laser therapy before exercise could significantly increase elbow flexion times (26). In a study in 2014 by Felissimo et al. in 22 male athletes, the pre-exercise laser did not make any significant differences in improving strength (27).

Perceived fatigue, which was assessed subjectively, did not show a significant change between the first and seco $^{\text {nd }}$ sessions in the placebo group, while in the treatment group, the difference was significant. Also, the perceived excretion in the $2^{\text {nd }}$ session decreased significantly in the treatment group compared with the placebo group. This finding shows that laser therapy can reduce muscular fatigue.

In a study in 2015 by Ferraressi et al., a laser at $630 \mathrm{~nm}$ and $850 \mathrm{~nm}$ wavelengths were applied on rats' lower limb. It was shown that a low-level laser could increase the mitochondrial content of muscles and delay exhaustion time. (28). In a systematic review in 2013 by Bursa et al. in 10 RCTs, they showed laser could delay the beginning of fatigue (29).

In a 2018 study by Manfreddiny et al. on 20 male cyclists, pre-exercise laser-accelerated oxygen delivery to mitochondria, especially in type 2 fibers, caused an increase in maximal $\mathrm{O}_{2}$ uptake, accelerated ATP synthesis, and delayed exhaustion time (30). However, in a study done by Alva et al. in 2014 on 18 sedentary subjects, it was shown that laser therapy before exercise test improves the immediate performance but has no effect on increasing time to exhaustion
(31). In a meta-analysis done in 2016 by Nampo et al. on 15 studies, the laser has not been effective in reducing DOMS in most studies. The authors suggested that laser therapy with a wavelength between $630-850 \mathrm{~nm}$ probably does not affect the management of musculoskeletal injuries (17).

Delayed onset muscle soreness, assessed subjectively 24 hours after the exercise session, in the placebo group did not show any significant differences between the first and second sessions. However, in the treatment group, the difference between the first and seco $^{\text {nd }}$ sessions was significant. Also, in the second session, DOMS in the treatment group decreased significantly compared to the placebo group. These findings show that laser can decrease exercise-induced muscle injury.

In a study by Antonialy et al. in 2014 in 40 healthy non-athlete males, a laser at $905 \mathrm{~nm}, 670$ $\mathrm{nm}$, and $875 \mathrm{~nm}$ reduced DOMS significantly (26, 32, 33). However, in a meta-analysis in 2016 on 15 RCTs, done by Nampo et al., in most studies, the appliance of laser could not have any effect on reducing DOMS (17).

Although these studies are performed in a wide range of wavelengths, therapeutic protocols, and different groups of participants and the results are very different, most of the authors suggest laser is useful in the improvement of athletic performance and musculoskeletal injury prevention, and the findings of this study are under this point of view.

\section{CONCLUSION}

Supporting our hypothesis, we found that laser therapy at $810 \mathrm{~nm}$ wavelength and $60 \mathrm{mw}$ power before exercise training may improve muscular performance and reduce muscle soreness and fatigue. However, more studies are necessary for athlete participants, with more extended followup periods and more exercise sessions. Future studies should evaluate muscular and neural activity in response to laser therapy precisely by EMG and NCV tests. In the current study, our subjects were selected from the sedentary population. Therefore, generalizing the results to athletes requires further studies.

\section{APPLICABLE REMARKS}

- low-level laser therapy may improve muscular strength and endurance and reduce fatigue and muscle soreness among young adults. 
CONFLICT OF INTEREST

There was no Conflict of interest.

\section{ACKNOWLEDGMENTS}

The authors are thankful for Bahar Hassanpur for helping in the statistical analysis.

\section{REFRENCES}

1. Hopkins JT, McLoda TA, Seegmiller JG, Baxter GD. Low-level laser therapy facilitates superficial wound healing in humans: a triple-blind, sham-controlled study. J Athl Train. 2004;39(3):223-229.

2. Bjordal JM, Lopes-Martins RA, Joensen J, Couppe C, Ljunggren AE, Stergioulas A, et al. A systematic review with procedural assessments and meta-analysis of low level laser therapy in lateral elbow tendinopathy (tennis elbow). BMC Musculoskelet Disord. 2008;9:75. doi: 10.1186/1471-2474-9-75 pmid: 18510742

3. Amorim JC, de Sousa GR, de Barros Silveira L, Prates RA, Pinotti M, Ribeiro MS. Clinical study of the gingiva healing after gingivectomy and low-level laser therapy. Photomed Laser Surg. 2006;24(5):588594. doi: 10.1089/pho.2006.24.588 pmid: 17069488

4. Maiya GA, Kumar P, Rao L. Effect of low intensity helium-neon (He-Ne) laser irradiation on diabetic wound healing dynamics. Photomed Laser Surg. 2005;23(2):187-190. doi: 10.1089/pho.2005.23.187 pmid: 15910184

5. Posten W, Wrone DA, Dover JS, Arndt KA, Silapunt S, Alam M. Low-level laser therapy for wound healing: mechanism and efficacy. Dermatol Surg. 2005;31(3):334-340. doi: 10.1111/j.15244725.2005.31086 pmid: 15841638

6. Chang WD, Wu JH, Wang HJ, Jiang JA. Therapeutic outcomes of low-level laser therapy for closed bone fracture in the human wrist and hand. Photomed Laser Surg. 2014;32(4):212-218. doi: 10.1089/pho.2012.3398 pmid: 24649935

7. Kazem Shakouri S, Soleimanpour J, Salekzamani Y, Oskuie MR. Effect of low-level laser therapy on the fracture healing process. Lasers Med Sci. 2010;25(1):73-77. doi: 10.1007/s10103-009-0670-7 pmid: 19399356

8. Silva Junior AN, Pinheiro AL, Oliveira MG, Weismann R, Ramalho LM, Nicolau RA. Computerized morphometric assessment of the effect of low-level laser therapy on bone repair: an experimental animal study. J Clin Laser Med Surg. 2002;20(2):83-87. doi: 10.1089/104454702753768061 pmid: 12017432

9. Rochkind S, Vogler I, Barr-Nea L. Spinal cord response to laser treatment of injured peripheral nerve. Spine (Phila Pa 1976). 1990;15(1):6-10. doi: 10.1097/00007632-199001000-00003 pmid: 2326702

10. Albarracin R, Eells J, Valter K. Photobiomodulation protects the retina from light-induced photoreceptor degeneration. Invest Ophthalmol Vis Sci. 2011;52(6):3582-3592. doi: 10.1167/iovs.10-6664 pmid: 21421867

11. Schiffer F, Johnston AL, Ravichandran C, Polcari A, Teicher MH, Webb RH, et al. Psychological benefits 2 and 4 weeks after a single treatment with near infrared light to the forehead: a pilot study of 10 patients with major depression and anxiety. Behav Brain Funct. 2009;5:46. doi: 10.1186/1744-90815-46 pmid: 19995444

12.Uozumi Y, Nawashiro H, Sato S, Kawauchi S, Shima K, Kikuchi M. Targeted increase in cerebral blood flow by transcranial near-infrared laser irradiation. Lasers Surg Med. 2010;42(6):566-576. doi: 10.1002/1sm.20938 pmid: 20662034

13.Lapchak PA, Han MK, Salgado KF, Streeter J, Zivin JA. Safety profile of transcranial near-infrared laser therapy administered in combination with thrombolytic therapy to embolized rabbits. Stroke. 2008;39(11):3073-3078. doi: 10.1161/STROKEAHA.108.516393 pmid: 18687999

14.Zivin JA, Albers GW, Bornstein N, Chippendale T, Dahlof B, Devlin T, et al. Effectiveness and safety of transcranial laser therapy for acute ischemic stroke. Stroke. 2009;40(4):1359-1364. doi: 10.1161/STROKEAHA.109.547547 pmid: 19233936

15. Vanin AA, Verhagen E, Barboza SD, Costa LOP, Leal-Junior ECP. Photobiomodulation therapy for the improvement of muscular performance and reduction of muscular fatigue associated with exercise in healthy people: a systematic review and meta-analysis. Lasers Med Sci. 2018;33(1):181-214. doi: 10.1007/s10103-017-2368-6 pmid: 29090398 
16. Baroni BM, Rodrigues R, Freire BB, Franke Rde A, Geremia JM, Vaz MA. Effect of low-level laser therapy on muscle adaptation to knee extensor eccentric training. Eur J Appl Physiol. 2015;115(3):639647. doi: 10.1007/s00421-014-3055-y pmid: 25417170

17. Nampo FK, Cavalheri V, Ramos Sde P, Camargo EA. Effect of low-level phototherapy on delayed onset muscle soreness: a systematic review and meta-analysis. Lasers Med Sci. 2016;31(1):165-177. doi: 10.1007/s10103-015-1832-4 pmid: 26563953

18.Leal-Junior EC, Vanin AA, Miranda EF, de Carvalho Pde T, Dal Corso S, Bjordal JM. Effect of phototherapy (low-level laser therapy and light-emitting diode therapy) on exercise performance and markers of exercise recovery: a systematic review with meta-analysis. Lasers Med Sci. 2015;30(2):925939. doi: 10.1007/s10103-013-1465-4 pmid: 24249354

19. Bublitz C, Renno AC, Ramos RS, Assis L, Sellera CA, Trimer R, et al. Acute effects of low-level laser therapy irradiation on blood lactate and muscle fatigue perception in hospitalized patients with heart failure-a pilot study. Lasers Med Sci. 2016;31(6):1203-1209. doi: 10.1007/s10103-016-1965-0 pmid: 27250713

20.de Paiva PR, Tomazoni SS, Johnson DS, Vanin AA, Albuquerque-Pontes GM, Machado CD, et al. Photobiomodulation therapy (PBMT) and/or cryotherapy in skeletal muscle restitution, what is better? A randomized, double-blinded, placebo-controlled clinical trial. Lasers Med Sci. 2016;31(9):1925-1933. doi: 10.1007/s10103-016-2071-z pmid: 27624781

21. Miranda EF, Tomazoni SS, de Paiva PRV, Pinto HD, Smith D, Santos LA, et al. When is the best moment to apply photobiomodulation therapy (PBMT) when associated to a treadmill endurance-training program? A randomized, triple-blinded, placebo-controlled clinical trial. Lasers Med Sci. 2018;33(4):719-727. doi: 10.1007/s10103-017-2396-2 pmid: 29185134

22. Toma RL, Oliveira MX, Renno ACM, Laakso EL. Photobiomodulation (PBM) therapy at $904 \mathrm{~nm}$ mitigates effects of exercise-induced skeletal muscle fatigue in young women. Lasers Med Sci. 2018;33(6):1197-1205. doi: 10.1007/s10103-018-2454-4 pmid: 29455305

23. Kakihata CM, Malanotte JA, Higa JY, Errero TK, Balbo SL, Bertolini GR. Influence of low-level laser therapy on vertical jump in sedentary individuals. Einstein (Sao Paulo). 2015;13(1):41-46. doi: 10.1590/S1679-45082015AO3243 pmid: 25993067

24.Ferraresi C, Beltrame T, Fabrizzi F, do Nascimento ES, Karsten M, Francisco Cde O, et al. Muscular pre-conditioning using light-emitting diode therapy (LEDT) for high-intensity exercise: a randomized double-blind placebo-controlled trial with a single elite runner. Physiother Theory Pract. 2015;31(5):354-361. doi: 10.3109/09593985.2014.1003118 pmid: 25585514

25.Ferraresi C, Huang YY, Hamblin MR. Photobiomodulation in human muscle tissue: an advantage in sports performance? J Biophotonics. 2016;9(11-12):1273-1299. doi: 10.1002/jbio.201600176 pmid: 27874264

26.Leal Junior EC, Lopes-Martins RA, Frigo L, De Marchi T, Rossi RP, de Godoi V, et al. Effects of lowlevel laser therapy (LLLT) in the development of exercise-induced skeletal muscle fatigue and changes in biochemical markers related to postexercise recovery. J Orthop Sports Phys Ther. 2010;40(8):524532. doi: 10.2519/jospt.2010.3294 pmid: 20436237

27.Felismino AS, Costa EC, Aoki MS, Ferraresi C, de Araujo Moura Lemos TM, de Brito Vieira WH. Effect of low-level laser therapy $(808 \mathrm{~nm})$ on markers of muscle damage: a randomized double-blind placebo-controlled trial. Lasers Med Sci. 2014;29(3):933-938. doi: 10.1007/s10103-013-1430-2 pmid: 24005882

28.Ferraresi C, de Sousa MV, Huang YY, Bagnato VS, Parizotto NA, Hamblin MR. Time response of increases in ATP and muscle resistance to fatigue after low-level laser (light) therapy (LLLT) in mice. Lasers Med Sci. 2015;30(4):1259-1267. doi: 10.1007/s10103-015-1723-8 pmid: 25700769

29. Borsa PA, Larkin KA, True JM. Does phototherapy enhance skeletal muscle contractile function and postexercise recovery? A systematic review. J Athl Train. 2013;48(1):57-67. doi: 10.4085/1062-605048.1.12 pmid: 23672326

30.Lanferdini FJ, Kruger RL, Baroni BM, Lazzari C, Figueiredo P, Reischak-Oliveira A, et al. Low-level laser therapy improves the VO2 kinetics in competitive cyclists. Lasers Med Sci. 2018;33(3):453-460. doi: 10.1007/s10103-017-2347-y pmid: 29119418 
31.da Silva Alves MA, Pinfildi CE, Neto LN, Lourenco RP, de Azevedo PH, Dourado VZ. Acute effects of low-level laser therapy on physiologic and electromyographic responses to the cardiopulmonary exercise testing in healthy untrained adults. Lasers Med Sci. 2014;29(6):1945-1951. doi: 10.1007/s10103-0141595-3 pmid: 24925070

32. Antonialli FC, De Marchi T, Tomazoni SS, Vanin AA, dos Santos Grandinetti V, de Paiva PR, et al. Phototherapy in skeletal muscle performance and recovery after exercise: effect of combination of superpulsed laser and light-emitting diodes. Lasers Med Sci. 2014;29(6):1967-1976. doi: 10.1007/s10103014-1611-7 pmid: 24942380

33. Vanin AA, Miranda EF, Machado CS, de Paiva PR, Albuquerque-Pontes GM, Casalechi HL, et al. What is the best moment to apply phototherapy when associated to a strength training program? A randomized, double-blinded, placebo-controlled trial : Phototherapy in association to strength training. Lasers Med Sci. 2016;31(8):1555-1564. doi: 10.1007/s10103-016-2015-7 pmid: 27371449 